\title{
LA INVESTIGACIÓN FILOSÓFICA
}

De nobis ipsis silemus, nos dice Kant citando a Bacon en el umbral de la Crítica de la razón pura, indicando la ignorancia que solemos tener de los problemas que conciernen a la interioridad del Yo que en el caso del magister prusiano es el funcionamiento del intelecto de análoga manera a como se presenta un fenómeno similar en cada proyección de la individualidad. Ya se trate de la res cogitans in genere o cualquiera de sus manifestaciones particulares el hecho es que "de nosotros mismos callamos" en tanto que de las otras cosas hablamos con abundancia. Esto mismo sucede con el investigador filosófico cuya tarea avócale directamente al estudio de sus problemas particulares, silenciando por regla general una cuestión que no es de poca monta, cual definir y expresar en qué consiste la investigación filosófica. Queriendo apartarnos un poco de este hábito silencioso decidimos intentar una breve caracterización del menester inquisitivo en los términos que supone la responsabilidad académica de hablar en la primera persona del singular, que es la instancia originaria en el primer estudio de la opinión filosófica.

\section{Historia y sistema}

Me parece que, por principio de cuentas, cabe distinguir dos grandes direcciones en la investigación filosófica, correspondiendo a las respectivas dimensiones en que se desenvuelve el filosofar, a saber: la histórica y la sistemática. Estas direcciones derivan a su vez de las categorías constitutivas en todo desarrollo cultural, como son la categoría diacrónica y la sincrónica. La primera representa la evolución del pensamiento en la secuencia histórica y la segunda en la integración sistemática y doctrinaria. Empero, grave error sería considerarlas abstractivamente, hablar de ellas como si fueran ajenas, cuando en realidad sucede lo contrario, se requieren ineludiblemente desde el momento que su compresencia recíproca es inexorable por la indole dinámica que corresponde a toda forma de realidad cultural y axiológica, más aún cuando se presenta con caracteres que denotan un altó grado de integración en el trabajo espiritual y específicamente filosófico.

Así pues, la categoría diacrónica se manifiesta en la evolución histórica, mientras la sincrónica se traduce en la organización sistemática; la historia es trayectoria diacrónica y el sistema es estructura sincrónica. No existe, por lo demás, una historia carente de sistema ni tampoco un sistema carente de historia, pues la historia es historia de los sistemas y el sistema es integración 
sistemática de la historia. Pero la aplicación de un punto de vista para filosofar implica necesariamente el distingo de ambos aspectos, que en alguna ocasión he comparado con los cortes anatómicos que se efectúan para estudiar los tejidos en el tallo de una planta; la apreciación histórica me parece como un corte longitudinal donde se observa el desarrollo diacrónico en un cierto tipo de problemas que se desplazan longitudinalmente, similar a los vasos por donde circula la savia vegetal, mientras el estudio sistemático sería parangonable al corte transversal, y pone de manifiesto la existencia de diversos tipos de problemas que coexisten en un momento dado, revelando esta multiplicidad de problemas a través del enfoque sincrónico que exhibe al sistema en su conjunto, tal como se observa en el corte que expone los vasos coexistentes a un cierto nivel del tallo.

La realidad filosófica es - valga la comparación- como el tronco de una planta intelectual, florecida en el terreno axiológico que denotan los valores de la cultura, y puede examinarse a partir de ambas modalidades direccionales, siempre y cuando se tenga en cuenta en cada caso la existencia de la categoría complementaria, o sea la sincrónica para el diacronismo y la diacrónica para el sincronismo: evolución longitudinal y coexistencia tranversal, respectivamente.

De este modo surge una tercera posibilidad para la investigación, que vendría a ser fundamental, pues frente a ella las otras quedarían en calidad de expresiones derivadas, como realizaciones parciales de un principio integrado. Me agradaría decir que la verdadera investigación filosófica debe ser diasincrónica, o sea, debe integrarse simultáneamente en las diversas etapas de su evolución y en las diferentes modalidades de su contenido. Sin embargo, no es posible llegar directamente al diasincronismo sin cursar sus dimensiones parciales y constitutivas. Así como es imposible estudiar fisiología sin efectuar previamente cortes anatómicos para enterarse de cómo funcionan las células y los tejidos, tampoco es factible llegar al estudio diasincrónico del pensamiento sin lograr el previo análisis que proporcionan las dos grandes formas de incidir en las funciones filosóficas, que son parciales con respecto a su modalidad integral, para conocer el resultado que se manifiesta en cada caso. Después de efectuar tales análisis previos, una posterior integración del conocimiento llega a ser indispensable para reconstruir la unidad del problema original, consistente en la obra doctrinaria tal como es producida en el seno de la historia.

Me parece que esta primera reflexión sobre la forma de plantear el problema y la definición del método a seguir, fundamenta las dos grandes modalidades que asume la investigación filosófica; el primer tipo, o sea la investigación histórica, quedaría establecido al adoptar finalidades interpretativas sobre las ideas producidas por los grandes pensadores que figuran en la historia, mientras el segundo, o sea la investigación sistemática, consistiría 
primordialmente en el desarrollo de las doctrinas propias que corresponden a cada investigador y que en el mejor de los casos deberán ostentar una estructura de sistema, pues la forma de erigir el pensamiento en su dimensión sincrónica es precisamente la del sistema, que equivale genéricamente a unidad del pensar frente a la multiplicidad de los problemas.

Debo aclarar que el término diasincrónico que propongo es equivalente a dialéctica, pues diasincronismo es tanto como dialéctica operante en espacio y tiempo, o sea la bidimensionalidad inherente a todos los fenómenos reales, y dentro de ellos, los hechos culturales. En cierto modo, el diasincronismo representa la suprema dialéctica por cuanto superiores son las dimensiones histórica y sistemática frente a las cuales se manifiestan las formas integrativas de rango parcial que traducen la mutua operabilidad de los elementos constituyentes, de modo que la evolución diacrónica, considerada en sí misma, es también dialéctica, así como la sincrónica reviste un carácter propio de evolutividad y dialecticidad, encontrando su complemento mutuo en los términos que he señalado. Análogamente surgen las respectivas modalidades de la investigación filosófica, que puede ser predominantemente histórica o sistemática, si bien ninguna de tales categorías puede aislarse por completo de la complementaria, por más radical que se suponga su realidad intrínseca y su operancia autónoma.

Concluiremos, pues, que es necesario tener en cuenta, al observar la evolución histórica, que se trata en última instancia de reconocer la integración histórica de los sistemas, o por el contrario, en el caso del sistema se tiene el desarrollo sistemático de la historia, requiriendo en cada caso la participación de sendas categorías dialécticas y complementarias. Este concepto de la filosofía y la investigación como modalidades del pensamiento dialéctico me parece de todo punto inexcepcional y en torno suyo gira el problema que nos ocupa; cada uno de los casos que señalo presenta ciertos problemas que es indispensable considerar. Principiemos por la forma diacrónica de manifestarse el pensamiento a través de la historia.

\section{Primera Parte: La Investigación Historica}

\section{Requisitos de la investigación}

En primer término, el conccpto de la investigación histórica se ha hecho consistir en una búsqueda de ideas ajenas $y$, por consiguiente, en la relativa carencia de las propias, tal como ha llegado a sostenerse en algunas apreciaciones sobre este tipo de investigación. Sobre ese punto de vista debemos observar que el investigador histórico estudia efectivamente ideas ajenas, pero ello no lo pone al margen de cualquier intervención activa de su parte; la faena historiográfica no puede ser absolutamente neutral en el sentido de no 
introducir elemento alguno en el material investigado, neutralidad que no se encuentra ni siquiera en el historiador más superficial, pues cualquier trabajo de este género pone en juego criterios de variada índole, principalmente selectivos e interpretativos, que auspician la participación activa del historiador y, por consiguiente, lo erigen en copartícipe de la historia, como una especie de asociado ex-officio a los autores cuyas ideas examina.

Para dar curso a mis apreciaciones en torno a este problema, quisiera establecer someramente cuáles son a mi juicio los requisitos de la investigación histórica y, por consiguiente, los pasos metódicos que se deben efectuar para llevarla a cabo. A tal efecto, diría que la faena historiográfica se realiza fundamentalmente en cinco etapas metodológicas, como son las siguientes:

a) Selección del campo histórico examinado, determinando su propia extensión y distinguiéndolo de los demás campos que puedan coexistir con él.

b) Ordenación del material; según la génesis evolutiva de las ideas en cada autor, lo cual determina la integración histórica propiamente dicha.

c) Jerarquización de acuerdo con un criterio que establece un orden de naturaleza axiomática, prosiguiendo los niveles de realización axiológica.

d) Interpretación de la secuencia evolutiva según los principios y niveles de la idea axiológica que se encuentra en cada sistema examinado.

e) Valoración o sea calificación de las doctrinas con el criterio valorativo que es la idea regulativa correspondiente a cada sector histórico-axiológico.

En los cinco puntos anteriores se contiene, a mi entender, el esquema básico de la investigación historiográfica y su adecuada aplicación entraña el correcto esquema formal de la historiografía científica, que admite además una gran variedad de modalidades interpretativas determinadas en todo caso por la índole especifica de cada territorio examinado.

La primera fase consiste en la selección del campo que va a ser investigado y se obtiene mediante la demarcación de un cierto ámbito doctrinario en la obra de un autor, seleccionando una parte de ella o bien dirigiéndose a un periodo más amplio que puede comprender dos o más pensadores, probablemente alguna escuela con varios de ellos o una cierta época, y en su extremo toda la historia de la filosofía occidental, como es el caso más frecuente en las obras que llevan ese título. La elección de la época, escuela o doctrina en uno o varios pensadores, e inclusive la historia por entero, es un acto que se reviste de capital importancia, pues con su sola definición queda automáticamente determinado el sentido de la investigación misma, desde el momento que fija su ámbito de operancia y, por consiguiente, el motivo de la propia investigación.

Esta fase constituye la aporética de la historiografía filosófica y, análogamente a como se observa con la aporética en general, el hecho de situar el problema que deba ser explicado, establece el sentido más amplio de la explicación, que en este caso se obtiene mediante la investigación histórica. 
Por otra parte, la selección puede llevarse a cabo aplicando dos puntos de vista capitales; el primero corresponde a la definición de dicho periodo inquisitivo en términos de una obra o una etapa determinada, mientras el segundo lleva a cabo la elección atendiendo a cierto tipo de conceptos que interesan particularmente al investigador. Esta dualidad de criterios ofrece por una parte la apreciación netamente diacrónica, o sea la evolución temporaria del pensamiento, por medio de la cual un determinado concepto adquiere diferentes acepciones, y por la otra permite la presentación de diferentes conceptos, tal como se encuentran en una cierta producción o en un determinado momento en la obra del pensador que se investiga. Como puede observarse, ambas posibilidades repercuten en las correspondientes maneras de entender el sentido funcional del método inquisitivo.

La segunda fase de la historiografía es la ordenación del material que debe ser historiado; la participación de dicha fase requiérese como una etapa necesaria del método inquisitivo para introducir un determinado orden de selección en un contexto que normalmente se redacta de acuerdo con las inspiraciones del momento, sin asumir el estricto rigor que supone la idea de homogeneidad en los materiales que integran un cuerpo de doctrina y casi nunca están incluidos en una misma obra sino encuéntranse repartidos en diferentes trabajos, muchas veces en mezcla con temas heterogéneos que deben ser analizados para lograr una cierta cohesión doctrinaria en el material expositivo. Este ordenamiento se efectúa casi siempre en función del criterio diacrónico que rige la evolución concreta del pensamiento y normalmente corresponde a la evolución del sentido teorético de los conceptos.

Ambos criterios son necesarios para obtener una imagen elocuente del acervo histórico que debe ser explicado para desenvolver un estudio monográfico y al mismo tiempo gradualmente ordenado en relación a la obra o periodo que se quiere investigar, atendiendo a que el material es casi siem: pre heterogéneo y para llevar a cabo su explicación requiérese una selección y ordenamiento de los temas que se desean incluir en la investigación. Como es sabido, los autores no siempre son muy precavidos en lo concerniente a la unidad temática de sus trabajos ni ofrecen la nitidez que sería deseable en su desarrollo para un mejor enfoque interpretativo.

Este hecho se observa principalmente en los filósofos llamados problemáticos, que cultivan de preferencia el género del ensayo, donde tienen cabida una diversidad de asuntos heterogéneos que, precisamente por su heterogeneidad, presentan un problema especiai al investigador, tanto más por cuanto la preocupación de sistema no es la más conspicua en el filósofo ensayista. En última instancia, existe siempre un apreciable coeficiente de ensayismo en todos los grandes filósofos debido a la originalidad de sus ideas, que constriñe a presentarlas callamum currentum, en la forma un tanto dispersa que es característica del ensayo. 
La tercera fase, o sea la jerarquización, es el resultado de la anterior, pues la ordenación de las ideas conduce necesariamente a reconocer determinada jerarquía en las mismas, de acuerdo con el principio admitido como norma fundamental que actúa en calidad de idea regulativa y criterio axiológico para desarrollar el sistema en general y cada uno de sus problemas particulares. El proceso de jerarquización implica una cierta posición exegética en cuya virtud se determina en cada caso la importancia que adquieren determinados principios en la integración del sistema. Esta fase debe acometerse destacando los aspectos más importantes de cada doctrina para revelar en ella ciertos puntos de inflexión que muestran definidamente el tránsito de una etapa a otra y de uno a otro problema en la integración del sistema que construye cada filósofo.

La fase de interpretación responde a la necesidad de explicar la génesis de las ideas en los términos que señalan los numerosos factores concurrentes a su producción y que son determinantes en la forma concreta como se manifiesta todo género de ideas en su acaecer histórico. Para llevar a cabo esta interpretación encontramos en primer término la evolución integrativa de las ideas que serán dispuestas según la lógica -o mejor dicho, la axiológica - interna de cada sistema, pues uno de los principios básicos que explican el devenir histórico es la tendencia en cierto modo conductista y en cierto modo teleológica que prosiguen los desarrollos teoréticos para llegar al coronamiento de cada noción sistemática; el aspecto teleológico se manifiesta de acuerdo con el desarrollo que reclama su basamento axiológico y, por otra parte, el aspecto conductista obedece a los factores extraaxiológicos o heterónomos que participan en su constitución real. Los términos de la interpretación señalan cuáles son los dos grandes hemisferios de toda doctrina, el lógico o axiológico, frente al extralógico o extraaxiológico, con lo cual se representa la evolución integrativa en el seno de la dialéctica autónoma y heterónoma que se manifiesta en cada sistema. Esta fase de interpretación prepara el advenimiento de la quinta y última que figura en el proceso de la investigación histórica.

Dicha fase consiste - según hemos visto- en la valoración emitida sobre la perspectiva integral en que es situada la doctrina para formular sobre ella un definido juicio de valor donde se exponga cuál fue su rendimiento esencial, la contribución más importante que ofreció a la historia filosófica, en qué forma se adecúa esa contribución al resto del sistema, cuáles de sus facetas específicas están de acuerdo con la parte medular del sistema, y cuáles, por el contrario, se contradicen con él. De esta apreciación derivan los criterios prospectivos y retrospectivos a los que nos referiremos después, por los cuales se ubica la doctrina en términos de una trayectoria superior donde ocupa cierta etapa de transición entre los sistemas anteriores y los posteriores, integrando el decurso evolutivo que observamos analíticamente en la sucesión de las épocas y las escuelas históricas. 
A su vez, el juicio valorativo puede remitirse en términos generales a una apreciación que exponga el conjunto del sistema en cada pensador, o derivar a cada una de sus obras, señalando la contribución que presenta en el conjunto del sistema, el momento evolutivo que significa en el mismo, llegando inclusive a la valoración de los pensamientos concretos que figuran como cumbres estelares del sistema y llegan en ocasiones a manifestarse en aforismos que pasan a la posteridad por su peculiar elocuencia y significación.

Indudablemente, con la judicación valorativa de los sistemas y sus pensamientos concretos, se cumple el designio más amplio de la investigación histórica, consistente en apreciar cada uno de los elementos que integran un sistema filosófico en función del valor que realiza todo el sistema, de los problemas que lo constituyen, de los pensamientos que lo consagran, para llegar finalmente a la reconstrucción integral de la historia, la cual se produce en última instancia sobre una ininterrumpida línea de continuidad, de donde la suprema investigación histórica seria la que agrupara en su integridad el decurso diacrónico del pensamiento, incorporando todos los sistemas desde todos los puntos de vista que sea posible poner en juego y que considero esencialmente como prospectivos, introspectivos y retrospectivos, cuyo efecto y significado expondré ulteriormente.

\section{Horizonte en la historiografia}

Observando en rigor, nunca se agota la posibilidad de emprender una revaloración de los autores más consagrados y las tesis tradicionalmente establecidas, pero a medida que transcurre el tiempo, dicha posibilidad disminuye en función de las investigaciones que se han desarrollado, produciendo el asentamiento natural de la convicción genérica donde reposa el núcleo general de la crítica histórica en términos de un consenso establecido por la investigación y sedimentado por el acuerdo del tiempo. Escasas son las teorías que puedan revolucionar actualmente las convicciones que se encuentran afirmadas en el tratadismo filosófico sobre el sentịdo de los temas capitales que figuran en la historia.

Me parece que la época de oro en la historiografía se registró durante el siglo pasado y la primera mitad del presente; a través de dicho lapso se constituyó como ciencia la historia de la filosofía y produjo sus mejores rendimientos, quedando -a mi juicio- un reducido margen de posibilidades para continuar con éxito esta labor; el horizonte es algo mayor en los tratamientos monográficos, o sea los que dirigen la tarea a determinados aspectos particulares que tal vez no se han puesto en claro con anterioridad, teniendo en cuenta principalmente las numerosas confusiones semánticas, conceptos evolutivos, desajustes dialécticos y aun contradicciones internas que por razón 
natural existen en los sistemas, cuya dinámica presenta un gran interés a la historiografía aunque me parece que las posibilidades de conocimiento e interpretación son relativamente pequeñas porque se han tratado en su mayor parte por conducto de la enorme cantidad de estudios generales y particulares que hasta ahora se han realizado a tal efecto.

De cualquier modo, también en el aspecto intensivo y evolutivo cabe señalar la mayor posibilidad actual a este género de investigación; el tratadismo monográfico de ciertos aspectos particulares en cada doctrina admite mayores exploraciones y llega a un alto nivel de especulación, produciendo algunos trabajos importantes donde suelen hallarse contribuciones especificas que ayudan a comprender el sentido dinámico de cada sistema. Tales estudios exigen un profundo conocimiento del tema que tratan y requieren la más aguda especialización del saber historiográfico para profundizar en el análisis diferencial de cada tema. Es posible que sólo por esta via puedan obtenerse en la actualidad algunas contribuciones significativas que arrojen nueva luz a la historia, superando el empleo de los criterios generales que se esgrimen comúnmente en aras del tratamiento especializado que encarna el virtuosismo profesional en este género de investigación.

Las variadas posibilidades de enfoque sobre los sistemas producidos, el gran volumen de trabajos que se han realizado y la abundante diversidad de temas que contienen, configura el volumen de trabajos que comentan las obras de la historia filosófica, un volumen mucho mayor que el de las obras comentadas. En efecto, la investigación dedicada a cualquiera de los grandes filósofos ocupa de hecho un espacio varias veces mayor que los escritos originales, aunque no por ello deba ser más importante, pues la originalidad de los filósofos creadores mantiene el primer orden de importancia en la valoración histórica. El mayor volumen que exhiben los comentarios obedece a la posibilidad que existe al emplear diversos puntos de vista analíticos que se utilizan para llevar a cabo el análisis histórico de las doctrinas, aplicando una múltiple correlación interna y externa con respecto a cada doctrina en el horizonte interpretativo de la investigación. La norma que rige la ordenación de dichos criterios es su sentido evolutivo y en él observo la presencia de tres grandes especies criteriológicas que corresponden a sendas posibilidades de correlación, a saber:

a) Criterios retrospectivos: señalan los antecedentes de una doctrina:

b) Criterios introspectivos: examinan la integración autónoma del sistema.

c) Criterios prospectivos: se refieren esencialmente a sus consecuencias.

Cada especie de criterios, o si se prefiere, cada fase o aspecto del triple criterio aplicado para llevar a efecto este análisis, se relaciona estrechamente con los demás y los supone en cuanto norma estructural de integración histórica. 
Así tenemos que la retrospección muestra cuáles fueron los pasos genéticos en la producción del sistema, o sean los supuestos antelativos en su formación previa, las influencias teoréticas y fácticas que lo determinan, etc.

La introspección exhibe el decurso evolutivo propiamente dicho en el desarrollo de las ideas características de la postura correspondiente.

La prospección indica las derivaciones ulteriores de cada doctrina, o sea las consecuencias que emanan de ella en los sistemas construidos con posterioridad.

Un trabajo que pueda considerarse completo, o cuando menos suficientemente explicativo y documentado en relación a cualquier tema monográfico, deberá atender necesariamente a esas tres dimensiones analíticas, pues si alguna quedara ausente se produciría grave deficiencia en la consiguiente concepción y explicación del sistema.

Esto es lo que se observa en el estudio cuyo objeto consiste en explicar un tema determinado, para lo cual debe necesariamente acudir a los antecedentes que sirven como punto de partida - retrospección-, también a los caracteres implícitos y logros obtenidos por la misma -introspección-y, por último, a las consecuencias que derivan del sistema - prospección-. Si faltara alguno de tales momentos históricos, el examen quedaría insuficiente en sus apreciaciones y defectiva la investigación realizada.

Resulta obvio que un estudio sobre Hegel -por ejemplo- deberá partir de los antecedentes kantianos que lo preceden y en gran parte definen el sentido de su doctrina, construida básicamente en torno a la "idea de la idea"; supondrá asimismo el aspecto esencial y contributivo del sistema hegeliano, que radica en la metafísica absolutista del espíritu histórico, para senalar finalmente las abundantes consecuencias que se reflejan en posturas ulteriores como el materialismo, el historicismo, etc., las cuales prosiguen la enseñanza dialéctica de la historia y la aceptan como contribución esencial de Hegel.

\section{SEgunda Parte: La Investigación Sistemática}

\section{Filosofía como axiologia}

En este punto llego al segundo tipo de investigación que me ocupa, o sea la investigación sistemática; se relaciona directamente con la postura sustentada por el investigador y la formación cultural que tiene como supuesto previo al desempeño de la tarea filosófica. El concepto que sóstengo considera a la filosofía fundamentalmente como axiología, o sea el sistema de los valores. La trascendental importancia que reviste la postura profesada para el logro de la investigación obedece al inexorable paralelismo entre una y otra, o sea entre la postura sostenida y la forma de investigar.

El problema que considero más importante y al mismo tiempo el más 
difícil de resolver, radica en la analogía estructural que se debe manifestar en los diferentes renglones culturales atendiendo a los valores que realiza cada uno; dicha analogía está determinada por el esquema arquitectónico de la cultura y constituye el basamento primario del filosofar, pues en él se apoya la posibilidad orgánica de llevar a cabo un trabajo sistemático de investigación y verificarlo adecuadamente en la cultura misma. No creo que sea posible consumar una investigación fecunda sobre un problema determinado, si no le apoya el concepto de su estructura axiológica, señalando ipso facto la analogía estructural y por consiguiente conceptual y metodológica, en las diferentes ramas de la cultura, así como la organización que priva en cada una.

Esta convicción explica mi variado interés sobre los contenidos culturales y la convicción que abrigo en el sentido de que solamente puede llegarse al núcleo central y más fecundo de la filosofía cuando se dispone de un amplio sustento cultural, una formación extensa que proporcione el sólido planteamiento de los problemas a partir de la realización axiológica, que es al mismo tiempo la manifestación objetiva del espíritu. El concepto de los valores representa el criterio que permite abordar la tarea interpretativa y valorativa de la cultura después de haber asimilado, aunque sea en el mínimo requerible, sus disciplinas fundamentales.

La razón para estipular el firme interés por el basamento cultural, consiste en penetrar los estratos de las disciplinas respectivas mediante sus proyecciones en la filosofía para obtener la noción sistemática de los valores que realiza cada una, así como del esquema categorial que se manifiesta en todos los valores como denominador genérico de la expresión espiritual. Ésta es la convicción que aflora en mis trabajos y que se podría formular diciendo: filosofía es axiologia.

Pero además, la cultura es también axiología en cuanto realización concreta de los valores subyacentes en las finalidades de la tarea cultural; de ahí la razón que me hace considerar a toda filosofía como una filosofía de la cultura, en la doble acepción que reporta este concepto, a saber, como filosoffa surgida en una época y sobre una dimensión coetánea de la cultura, al mismo tiempo como filosofía que se ocupa de ella en todas sus tareas, es decir, como la reflexión trascendental que toma a la cultura como objeto de múltiple estudio e insustituible objetividad. Ésta es la base para llevar a cabo la investigación sistemática, o si se prefiere, es la convicción personal que abrigo al desenvolver la misma, de acuerdo con la postura axiológica que profeso.

Así pues, la filosofía equivale a una axiología de la cultura; todo el filosofar gira para mí en torno a una doble acepción que relaciona, por una parte, a la filosofía como producto del medio cultural y por la otra establece como criterio de valoración el hecho de la cultura misma. De este modo 
considero a la historia filosófica como una integración gradual y paulatina que hace evolucionar el concepto expuesto de acuerdo a la tónica cultural de cada periodo y de cada nación. Reconozco los sistemas que se han producido en la historia como válidos, en la medida que realizan dicha función axiológica, la cual encuentro opuesta a las especulaciones metafísicas de toda especie, que se distinguen en su multívoca presentación por su carácter abs. tractivo e impiden su verificación en la experiencia o renuncian a ella al pretender trascenderla para remontarse en aras de la imaginación personal.

Pero aún en este caso, los sistemas metafísicos adquieren algún sentido en la medida en que sirven de preparación a ciertos conceptos ulteriores que se emitirán por la vía objetiva, asumiendo por tal motivo un cariz científico, al contar con el necesario fundamento del cual carece la metafísica; incluso, podríamos decir que desempeña una función como herramienta potencial del intelecto, como laboratorio experimental de las teorias, aunque ciertamente no tendrán vigencia sino al advenimiento de la objetividad, es decir, cuando superen el estado de especulaciones metafísicas y se conviertan en teorfas científicas y culturales. Comprendo, sin embargo, que los simpatizadores del desenfado metafísico - que afortunadamente cada día son menos- pensarán lo contrario y probablemente la filosofía tenga valor para ellos en la medida en que es producto de la libre especulación, en cuyo caso sujetarse a la experiencia equivale al intolerable aherrojamiento del pensar tan alegremente ejercido por los metafísicos.

Otros profesos, como pueden ser los positivistas, los materialistas, los historicistas, los individualistas, los escépticos, los dogmáticos, etc., abrigan en cada caso una postura por considerarla válida y rechazan a las demás, deformando a la historia con esta unilateralidad de criterio en función de su creencia particular, de donde el carácter subjetivo que asume frecuentemente la investigación.

\section{Influencia de la posiura}

Así pues, el concepto de la investigación filosófica, entendida en su acepción dinámica y sistemática, está íntimamente ligado a la idea que el investigador profesa de la filosofía misma. El concepto de las relaciones entre filosofar $\mathrm{e}$ investigar influye principalmente si de investigación sistemática se trata, y en menor grado, si la investigación es histórica. No podría ser de otro modo, pues lo que hace el investigador es en último término filosofar sobre el sistema o la historia de la filosofía; no concibo ciertamente - como ya lo expresé al hablar de la investigación histórica - ninguna posición inquisitiva que elimine la participación activa del investigador para dejarlo en calidad de observador pasivo, en cuyo caso dejaría de ser ipso facto investigador para convertirse en simple lector. 
A tal grado es determinante la índole de la postura filosófica que cualquier investigación suficientemente desarrollada la confronta. No es concebible una investigación anómica y pasiva que se limitara a percibir tesis ajenas y contemplar acontecimientos como si transcurrieran en un desfile espectante, renunciando de antemano a cualquier intervención normativa y calificativa sobre los mismos, prescindiendo de todo juicio de valor que implique una adopción de postura y sistema, así como de las funciones inherentes a la investigación, tanto histórica como sistemática: selección y ordenamiento de materiales, empleo de un criterio para juzgar e interpretar, y lo que resulta de todo ello inevitable, una confronta entre los problemas que se examinan y el criterio utilizado para juzgarlos, cuestión esta última de la que no siempre es plenamente consciente el investigador.

Con todo lo anterior, queda en pie la conclusión expuesta: resulta imposible evitar que la investigación filosófica quede matizada por el criterio del investigador y refleje en un grado variable sus convicciones; este grado será menor tratándose de investigación histórica y mayor cuando es sistemática, pues en este último caso tiene la más amplia posibilidad de expresar sus ideas, mientras que en el primero debe sujetarse en la mayor medida al contexto examinado.

La exploración de las relaciones que se establecen entre la postura sustentada y sus consecuencias en la investigación, expone la raíz que manifiestan todos los aspectos de la filosofía con respecto a la cultura, lo cual lleva a reconocer que la aplicación concreta de la filosofía debe registrarse en el campo de la cultura misma. En virtud de esta relación -prosiguiendo siempre en mi punto de vista - el filósofo reconoce como punto de partida y de verificación al hecho cultural; lo primero, porque se inicia mediante la observación del acaecer evolutivo en la experiencia, en las variadas manifestaciones del devenir histórico, y lo segundo, porque las teorias filosóficas, referidas como se encuentran a los hechos de la cultura, deben encontrar su verificación en la misma y propugnar además por un continuo resguardo del proceso histórico mediante el develamiento de los valores que en él se encuentran. Con ello reafírmase el sentido clásico de la filosofía, expuesto en el doble motivo de que la tarea filosófica está enclavada en un marco de acción cultural, donde tiene su origen y criterio de verificación.

Éste es el caso del hombre de ciencia que se preocupa por investigar los problemas lógicos de su disciplina, los cuales entrañan las formas básicas del pensamiento; el caso del artista que hace lo propio con las cuestiones estéticas del arte, preguntando en qué radica la belleza de las obras; del jurista que analiza los fundamentos éticos del derecho y va más allá de su aplicación formalista para exponer su basamento moral, y así sucesivamente.

En cada uno de los sectores culturales se localiza un problema radicado en el contenido axiológico de la cultura, o sea el valor subyacente en cada 
manifestación del espíritu, cuyo develamiento implica, en el sentido más auténtico, el acto de producir una investigación consistente en retomar los problemas de la experiencia cultural y construir el edificio filosófico en su más puro sentido sistemático, mediante la reflexión que se origina directamente en el estudio de los hechos y los valores. Esta conclusión va de acuerdo - según dije antes - con el concepto que profeso de la filosolía, pues al no concebir su manifestación abstracta o lucubrativa, el camino para una filosofía concreta y al mismo tiempo creadora, no puede ser otro que el análisis axiológico de la existencia. Éste es el concepto más estricto, la tarea más depurada que reconozco para la investigación sistemática de la filosofía, diría la única, pues según el mismo criterio no existe otra que pueda garantizar los irrevocables requisitos que deben fijarse a la investigación sistemática, a saber:

a) Concreción para definir el objeto real que sea problema propio de la filosofía. sófica.

b) Unidad que reafirme la estructuración sistemática de la tarea filo-

c) Trascendentalidad que excluya las duplicaciones en el planteamiento axiológico.

\section{La filosofía como ciencia}

Quiero permanecer en el desarrollo de este propósito lo más objetivo que sea dable, incondicionalmente objetivo querría decir, ya que no es posible mantener una postura de investigación basada en creencias y opiniones personales; por el contrario, se refuerza cada día la convicción de objetividad en la cual se funda el concepto intelectivo de toda ciencia. Sin embargo, me parece que no es posible hablar de la investigación filosófica sin referirla directamente a la postura que se sustenta, la cual en todo caso debe presentarse como objetiva, pues cada quien prosigue la faena inquisitiva atendiendo a sus convicciones teoréticas y las ampara en ideas básicas de objetividad, pero permanece atado al cordón umbilical del fuero subjetivo en una proporción que resulta difícil precisar a priori, bordeando el riesgo de la unilateralidad doctrinaria que se presenta de hecho en todo sistema, obedeciendo a razones que son, precisamente, razones de hecho.

¿Hasta qué grado puede variar el concepto genérico de la investigación filosofica, teniendo en cuenta la divergencia de criterio que impera en los pensadores y los sistemas? Tal es probablemente uno de los puntos más importantes que deben resolverse tendiendo en todo caso a producir un concepto científico de la investigación, o si se prefiere $e l$ concepto de la investigación filosófica considerada como ciencia, para lo cual se requiere establecer 
algunas condiciones que figuran como postulados básicos en la definición de toda ciencia. ¿Cuáles son estos axiomas, tratándose de la filosofía?

La dificultad para precisarlos estriba en un hecho evidente como es que la filosofia misma no se erigió desde sus comienzos como una ciencia, sino en calidad de una meditación más o menos esporádica y en cierto modo informal. El antiguo concepto de filosofía, que en buena parte se mantiene aún, la considera como reflexión universal, tendiente a abordar problemas de toda especie, tal vez como panacea para resolver los problemas angustiosos del espíritu o para especular en cualquier momento sobre cualquier tema, como en efecto sucedió durante una larga etapa de su historia, la mayor parte podríamos decir, pues se extiende desde sus comienzos en las antiguas civilizaciones por la gran mayoría de su duración cronológica. En toda la prolongada era anterior al moderno racionalismo que encuentra su primer apogeo en Descartes, la filosofía se desenvuelve como una meditación un tanto esporádica e imprecisa en sus linderos con otras disciplinas, lo cual no significa que necesariamente hubiera producido falsedades, aunque muchas de ellas figuran en teorías más o menos consagradas del campo metafísico; pero en todo caso, el método empleado fue circunstancial y de ningún modo se reconoce, a lo largo de esa dilatada prehistoria, la sistemática precisión e indeclinable objetividad que es inherente al pensamiento científico.

La idea de cientificidad en filosofía tiene significativos antecedentes en Sócrates y Platón, pero aflora hasta el racionalismo del siglo xvir, a imagen y semejanza de los desarrollos matemáticos que por aquel entonces cautivaban a los adalides de la intelectualidad; en esa época se produjo el primer gran acercamiento entre el pensamiento filosófico y el científico en los términos de una deducción trascendental, al reconocer expresamente que todo el pretérito filosófico debía ser puesto entre paréntesis para dar paso al nuevo estilo de filosofar que debería ser, a la spinoziana manera, more geometrico.

¿Qué significa entonces la filosofía como ciencia? ¿Cuáles son los axiomas que la caracterizan inequívocamente? $\mathrm{La}$ respuesta que puedo proponer a tan delicado interrogante contiene una primera fase que caracteriza a la filosofía como meditación general, la más general que produce el intelecto en cada época de la cultura. Dentro de esta generalidad se desenvuelve la prolongada fase prehistórica que alberga una meditación demasiado imprecisa y falta de verificación, aunque no obstante, reconoce un problema definido como objeto de sus reflexiones. De alguna manera se manifiestan las primeras etapas de toda ciencia y no olvidemos que ninguna de las disciplinas que actualmente se presentan en un alto grado de evolución tuvo en su origen ese nivel de adelanto, sino formas y conceptos más o menos rudimentarios que se fueron presentando cada vez más elaborados, a medida que avanzaron en la dirección prospectiva de la historia. En cambio, si observamos retrospectivamente, entroncamos con las creencias mágicas e irracionales 
que representan la protohistoria de la ciencia y la filosofía, donde sin embargo se contiene el primer planteamiento rudimentario de sus problemas. Este señalamiento representa algo más que una alusión incidental sobre el origen histórico de la filosofía, pues su etapa de integración se extiende durante un periodo sumamente prolongado, en el cual la cientificidad filosófica se difunde en una multitud de conocimientos fragmentarios, pero cuya fragmentariedad no les impide presentarse como verdades efectivas.

Preguntemos, para concluir este aspecto de nuestras meditaciones, si puede considerarse a la filosofía, y por consiguiente a la investigación filosófica, en calidad de ciencia, tema éste que se ha discutido en numerosas ocasiones y sin embargo permanece todavía en la penumbra de la indecisión, no porque el tema en sí resulte insoluble, sino por efecto de un nocivo prejuicio que considera demasiado restringido el concepto de ciencia, confiriéndolo exclusivamente a las disciplinas de corte matemático-naturalista y excluyendo no sólo a la filosofía, sino también a las ciencias sociales, que deben aceptarse sin restricción en la calidad científica que les corresponde.

Así-tenemos que mientras para algunos la filosofía es ciencia, para otros no lo es; prefieren dejarla en el nebuloso dominio de la especulación, donde ha estado durante largos periodos. Existen ciertos sectores donde se desea que la filosofía quede investida por una libertad irrestricta, y en ellos ha llegado a creerse que tomarla por ciencia equivale en cierto modo a degradarla, pues se le restaría una libertad de imaginación y de concepción que en otra forma conserva. Aunque parezca mentira, opiniones de este jaez suelen presentarse todavía en ambientes reputados de la intelectualidad, y con gran desparpajo se condena a la pretensión de cientificidad como indigna del verdadero filósofo, que sería en esta metafísica noción el gran señor feudal del intelecto, propietario a su arbitrio de las ideas, su ejercicio y su expresión.

La respuesta a semejante problema, es decir, que la filosofía y por consiguiente la investigación sistemática sea o no una ciencia, depende lógicamente del concepto que se tenga de la ciencia misma; es cierto que al adoptar como noción genérica de la cientificidad la muy circunscrita de las ciencias matemático-naturales, habrá que eliminar desde luego a las disciplinas que no se conforman con ella y en primer término figura la filosofía. Empero, si extendemos la idea de ciencia a todo el ámbito conceptual que le corresponde, interpretándola en general como saber sistemático y objetivamente verificable, entonces la filosofía y la investigación adquieren pleno derecho para ostentar semejante calificativo. Para explicar por qué la más amplia acepción de ciencia debe exigir el carácter de sistema entendido lato sensu como organización unitaria y estructural del conocimiento, y no circunscriptamente como sistema deductivo-racionalista, recordemos que el propósito explicativo de la ciencia consiste nada menos y nada más que en la disciplina 
unitaria y sistemática del saber, en cuya sola virtud es posible coordinar un conjunto de conocimientos que proporcionan la explicación siempre relativa, aunque progresiva, de un objeto o problema.

De producirse lo contrario, o sea la falta de unidad y de sistema, el saber permanecería en calidad de conocimiento fragmentario, tal vez como sucede en la vida cotidiana o como se observa en ciertas filosofías anecdóticas y problemáticas, que no constituyen efectivamente una verdadera ciencia sino a lo más un conjunto de observaciones probablemente certeras, aunque desligadas entre sí, como sucede en los aforismos más o menos notables, pero al fin de cuentas inconexos, de la sabiduría popular, en las máximas sapientes y esporádicas que envuelven algunas expresiones de la "filosofia" cotidiana, un saber demasiado primitivo con respecto a la filosofía sistemática, o lo que equivale, a la filosofía y la investigación filosófica consideradas como ciencia.

\section{El método trascendental}

De acuerdo con lo que expuse antes, la postura que sustento -en paralelo a la dilatada corriente neocriticista- debe entenderse como un constante filosofar en paralelo a la cultura, esto es, como una reflexión axiológica, autónoma, permanente y trascendental. La investigación sistemática y creadora consiste nada menos y nada más que en filosofar, situarse frente al hecho cultural y exponer sus valores de realización, tanto genéricos como específicos, o sean los que efectúa en su ámbito común y los que produce en cada una de sus ramificaciones particulares. Este desempeño que adquiere la filosofía en el sistema conjunto de las disciplinas culturales para develar los valores subyacentes en cada una, proporciona la pauta para la faena inquisitiva que deriva de la filosofía considerada como axiología, en cuyo planteamiento radica el temario básico del filosofar. Por ello reafirmo una vez más: el problema esencial de la filosofía es el problema de los valores; acometerlo objetivamente es filosofar $\mathrm{y}$, a su vez, también investigar.

Veamos, pues, que la proyección creativa de la filosofía presupone una suerte de elementos que son cuando menos tan importantes como la erudición en las doctrinas filosóficas; me refiero al conocimiento de los hechos culturales que se deben explicar, sin los cuales no habria punto de apoyo para reflexionar sobre el fundamento de los mismos, obtenido en el orden de la axiología trascendental, ni verificar las doctrinas axiológicas de la cultura en el factum de la realidad misma.

La posibilidad de esta investigación creativa está en relación directa con el método que se emplee para filosofar, el cual consiste en poseer y ejercitar una dotación básica de principios generales que actúan como norma general de proyección para las doctrinas filosóficas y las ramas culturales, de manera que exista una correspondencia entre éstas y aquéllas. Ambos 
tipos de conocimientos se requieren para llevar a cabo la investigación axiológica fundamentante que es privativa de la filosofía. El ejercicio del método trascendental se desenvuelve en términos de valor e implica, por consiguiente, el sistema de las categorías axiológicas generales que definen la constitución estructural del valor y participan como condiciones previas en su caracterización, desde el momento que fijan los modos de ser y condicionan las conclusiones particulares que derivan de la filosofía como resultado de la fundamentación axiocultural que está avocada a realizar.

Actualmente no puede ya existir una filosofía nacida por generación espontánea, a la manera de la especulación metafísica al viejo estilo, sin tener a mano las exigencias generales de la metodología filosófica; ésta reclama el contacto con el sustrato material, el objeto de conocimiento representado en la cultura; en la filosofía que yo reconozco, el método trascendental, crítico o axiológico, significa el instrumento insustituible para llevar a cabo una investigación específica. La cultura es el punto de partida para producir la investigación y el método trascendental es el camino para obtenerla. Con ello se impone la necesidad de precisar cuál debe ser el método adecuado para investigar sistemáticamente en filosofía; sobre este punto quisiera decir algunas palabras.

En la investigación historiográfica, el método se erige fundamentalmente con los elementos requeridos para llevar a cabo la consulta dirigida sobre los problemas planteados en la temática de los sistemas anteriormente producidos; su objeto es proporcionar la interpretación que se confiere a dichos sistemas a título explicativo de su desarrollo diacrónico, implicando las diferentes fases que mencioné antes.

En cuanto al método de la investigación sistemática, ya expuse que tiene como antecedente la asimilación de las disciplinas culturales y admite como complemento integrativo el conocimiento de las teorías filosóficas que se han pronunciado sobre los diferentes territorios culturales que sirven de punto de partida y sustrato para la reflexión. Podemos sintetizar la operabilidad del método trascendental en dos condiciones o requisitos previos como son los siguientes:

a) Conocimiento de la cultura.

b) Conocimiento de la filosofía.

Sin el primero - conocimiento de la cultura - se carecería de la base requerida para establecer la perspectiva creadora, la tarea concreta y su verificación en la experiencia; sin el segundo - conocimiento de la filosofía- faltaría el antecedente que despeja el campo para ejercêr la reflexión, pues al faltar dicho antecedente conviértese la filosofía en un prolijo ensayar el pronunciamiento de hipótesis lucubrativas, o en el mero señalamiento empírico de la experiencia fragmentaria, que no permite arribar a conclusiones generales. Contrariamente a ambas posiciones defectivas, y superándolas definiti- 
vamente, el genuino método filosófico-sistemático permite ascender de la experiencia múltiple de los hechos culturales a la unidad explicativa del valor, y volver de ella al múltiple de la experiencia para verificar las tesis axiológicas, por lo cual consta dicho método fundamentalmente de las siguientes fases o momentos en su desarrollo:

a) Concreción de la multiplicidad fáctica en la experiencia cultural, para tomar noticia de su realidad, seleccionando y clasificando los campos de cada especie sobre criterios judicativos de homogeneidad axioteleologica.

b) Abstracción de la unidad teorética que se obtiene develando la estructura axiológica en cada sector de la cultura, asumiendo la función explicativa que corresponde a cada sector cultural cuyo valor explica su sentido.

c) Verificación de la unidad axiológica como función explicativa en el campo cultural del cual partió, comprobando la regularidad aplicativa del valor mediante la categoría teleológica universal y la categoría específica en cada rama de cultura.

Estas fases corresponden ineludiblemente a los momentos de realización de la filosofía sistemática y pertenecen al método trascendental, llamado aś en la escuela criticista el método filosófico, principalmente por la erección del segundo momento, que implica la ascensión trascendental a la unidad del valor, en el cual se encuentra la razón explicativa del problema planteado sobre la realidad de la experiencia cultural.

Es fácil percatarse de que este esquema corresponde en términos generales al método que puede emplearse en cualquier problema, pues el solo hecho de plantear un tema epistémico, o sea el que debe acometer objetivamente el pensamiento, reclama partir asimismo de una multiplicidad empírica en la cual se ubica, indagar a continuación la correspondiente unidad explicativa que remite a la hipótesis trascendental de carácter axiológico -el valor del conocimiento es la verdad- y verificarla en la experiencia para poder aceptarla como valiosa, esto es, como válida o verdadera.

Los tres momentos del método trascendental son indispensables para efectuar la investigación sistemática y no puede faltar ninguno de ellos; si quedara ausente el primero, se carecería del objeto que trata de explicar la investigación, puesto que en ese primer momento se plantea el problema cognoscitivo; si el segundo faltara, sería imposible obtener ningún principio fundamentante, ningún criterio general de valor, pues la ascensión trascendental a la hipótesis arroja la unidad regulativa que actúa como principio explicativo; a su vez, el tercer momento no puede faltar porque la teoria explicativa debe ser verificada en el campo de la multiplicidad aporética donde se situó el problema que es objeto de la explicación, con lo cual se implica la necesaria presencia del tercer momento, o sea la fase de verificación. 


\section{La filosofia concreta}

Quisiera referirme en la última parte de esta semblanza de la investigación sistemática, a lo que llamaré el ejercicio concreto de la filosofía, o sea la manera real de filosofar, tal como se manifiesta en el acto efectivo del pensamiento. Este ejercicio asume capital interés para precisar no solamente el desempeño que corresponde al filósofo en cuanto profesional de su materia, sino principalmente como hombre destinado a ejercitar el pensamiento en beneficio del medio que lo rodea, de la sociedad en que se desenvuelve $\mathrm{y}$, más ampliamente, del momento histórico que vive.

Esta consideración parte del supuesto de que debemos situarnos más allá, no solamente del dogmatismo particularista implicado en el ejercicio de una postura determinada, sino también del abstractismo que suele encontrarse en la profesión filosófica y la consiguiente indiferencia para los problemas positivos que acontecen en la realidad. Para apoyar esta convicción quiero remitirme una vez más a la idea ampliamente reconocida, en el sentido de que las posturas históricamente dadas no son una afloración evanescente del pensar, sino la expresión de un momento histórico a través de una problemática que deriva del estado imperante en la cultura y la realidad concreta de su tiempo.

Por ello, las doctrinas filosóficas asumen el papel de instrumentos inte: lectuales cuya forma y función se aplica a la problemática que les conviene, de análoga manera a como una herramienta en el taller se adapta a un trabajo determinado y sirve específicàmente para desempeñar tal menester; cada vez que se quiera ejecutar ese trabajo se recurrirá a dicha herramientá, cuya función no puede sustituir a las otras ni ser reemplazada ella misma por las demás, de acuerdo con el inalterable principio que vincula a la forma y la función de cada instrumento material o intelectual. Cada sistema filosófico responde a un problema concreto, obedece al método conducente a su explicación y realiza la función aporética para la cual fue concebida.

Por ello es por lo que la filosofía por una parte proviene y por la otra se aplica concretamente al ejercicio vivo de la reflexión, de donde resulta, en ese aspecto general, parangonable al oficio desempeñado por un operario en un taller, que lo es también el filósofo del intelecto, cuyo desenvolvimiento exige aplicar una forma de reflexión y una suerte especifica de método para cada tipo de problema abordado.

Cuando afirmamos, con Kant, que no se debe enseñar una filosofía sino a filosofar, podemos agregar "el oficio de filosofar", juntamente con las teorías eidéticas que constituyen el summum del pensamiento. Debería enseñarse el oficio, la práctica del pensar, el ejercicio vivo del intelecto, que está relacionado estrechamente con el tema de la investigación; én la medida de la herramienta intelectual que sé requiere como método parà su empleo. 
Hay un cierto aspecto de artesanía en este oficio intelectual y me parece que la convivencia directa y prolongada con un maestro es la mejor forma de asimilarla, tanto por la manera de ejercitar el intelecto como por las técnicas pragmáticas de investigar y redactar, que podrian aprenderse mejor directamente de un verdadero maestro, conviviendo con él en la faena cotidiana, un poco a la manera de los antiguos talleres artísticos del Renacimiento, cuya mejor réplica son las escuelas de trabajo en las modernas universidades.

A tal respecto, me parece que se observa actualmente en la filosofía mexicana una sensible decadencia con respecto al nivel existente hace todavía unos lustros, cuando florecieron generaciones tan conspicuas como la de Antonio Caso y sus contemporáneos, posteriormente la de sus discípulos, y más tarde una nueva generación que podríamos llamar de "los discípulos de los discípulos", que por aquel entonces representaban a la juventud filosófica y cuyo entusiasmo les llevó a agruparse en escuelas de trabajo, como fueron las de los neokantianos, los existencialistas, los neotomistas y los marxistas, o sean las cuatro principales tendencias en las que se reveló un marcado interés por la filosofía, traducido en un trabajo intensivo que, independientemente de su valor y rendimiento, exhibía cuando menos interés desbordante, cierta conciencia de grupo que de ningún modo era un solo gregarismo y que en la actualidad está disuelta, lo que a mi juicio es un síntoma decadente que relaciono con otros de pareja índole, como es el hecho depresivo de que en la actualidad no contemplemos una nueva generación de jóvenes que complemente a las anteriores y pueda suplirlas en el momento histórico que le corresponda para asumir el liderato de la vocación filosófica. Si esta deficitaria situación no encuentra un pronto remedio, todo hace augurar que la decadencia en tan importante rama de la cultura nacional se acentuará hasta el punto de no encontrar, siquiera en potencia, las personas que puedan cubrir los sitios de investigadores y catedráticos en filosofia.

Me parece que el panorama es igualmente depresivo en la provincia, pues no obstante los esfuerzos llevados a efecto para promover el desarrollo de la cultura filosófica, hasta el momento no se ha formado un solo profesional competente para la investigación y que esté ampliamente capacitado para ejercer la docencia filosófica en el nivel universitario. Sería muy conveniente que meditáramos un poco sobre tan angustioso problema, para determinar las causas que originan esta decadencia y, de ser posible, intentar alguna solución que pueda corregirla para beneficio directo de nuestra cultura filosófica, particularmente en el sector que nos ha ocupado en este comentario, o sea el de la investigación.

Miguel Bueno

Centro de Estudios filosóficos

Universidad Nacional Autónomia de México 